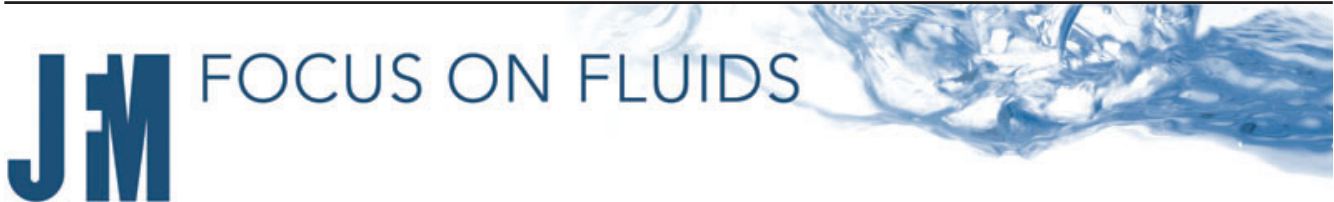

\section{Scale-invariant spins and tumbles in turbulence}

\author{
Varghese Mathai $\dagger$ \\ Department of Physics, University of Massachusetts, Amherst, MA 01003, USA
}

\begin{abstract}
A Lagrangian perspective has yielded many new insights in our quest to reveal the intricacies of turbulent flows. Much of this progress has been possible by following the trajectories of idealised, inertialess objects (tracers) traversing through the flow. Their spins and tumbles provide a glimpse into the underlying local velocity gradients of the turbulent field. While it is known that the spinning and tumbling rates of anisotropic particles are modified in turbulence - compared with those in a random flow field a quantitative explanation for this has remained elusive. Now, Pujara et al. (J. Fluid Mech., vol. 922, 2021, R6) have made an attempt to predict the split between spinning and tumbling rates by accessing the particle's alignment with the local vorticity. Their analysis of filtered turbulent fields reveals a Lagrangian scale invariance, whereby key quantities relating to the particle's rotational statistics are preserved from the dissipative to the integral scale.
\end{abstract}

Key words: particle/fluid flow, turbulent flows, isotropic turbulence

\section{Introduction}

Practically almost all of the turbulent flows around us contain tiny particles suspended in them. The movement of these particles as they are carried along seemingly erratic trajectories is central to our physical intuition of turbulent transport. Following their motion in a Lagrangian manner offers unique insights that cannot be perceived from the more traditional Eulerian viewpoint of the fluid velocity at fixed points in space (La Porta et al. 2001). Consider a tiny, inertialess, spherical object advected by a fluid flow. The trajectory it traces is identical to that of the surrounding fluid: adopting not just the local fluid velocity, but also the angular velocity (rotation rate) of the fluid. Yet, even as the particle tightly adheres to these movements, we miss out on a few crucial, defining features of the flow; the spherical particle is essentially immune to the local strain rate. Fortunately, this crucial ingredient of the flow can be probed if the particle were to morph into an ellipsoid. The picture of an elongated or flattened entity (rod-like or disk-like) advected by the turbulent field approximates quite nicely the shapes of bacteria

$\dagger$ Email address for correspondence: vmathai@umass.edu

(C) The Author(s), 2021. Published by Cambridge University Press. This is an Open Access article, distributed under the terms of the Creative Commons Attribution licence (http://creativecommons.org/ licenses/by/4.0/), which permits unrestricted re-use, distribution, and reproduction in any medium, provided the original work is properly cited. 


\section{Mathai}

and plankton in the turbulent oceans. Often, the behavioural responses of these organisms in natural environments are triggered by rotational motions, which arise as a result of the local velocity gradients in the fluid phase (Guasto, Rusconi \& Stocker 2012).

While it may appear simple to seed a turbulent flow with tiny ellipsoidal tracers and observe their kinematics, in practice, the tinier the particle the trickier it is to observe the rotations. Hence, the initial impetus in this field was certainly on the numerical side. The model equation governing the rotation of a small, non-inertial, ellipsoidal particle has been well within our grasp, thanks to the pioneering works of Jeffery (1922) and many others who verified its applicability (Voth \& Soldati 2017). The axisymmetric ellipsoid (spheroid) essentially rotates in response to two torques: one tending to make the particle adopt the same rotation as the surrounding fluid (like a spherical tracer), and the other aligning the particle's axes parallel to the principal axes of distortion of the fluid. A vast number of studies were launched over the past two decades, solving Jeffery's equation along Lagrangian fluid trajectories for many canonical fluid flows (Voth \& Soldati 2017). Shin \& Koch (2005) were one of the first to study the anisotropic particle dynamics using direct numerical simulations (DNS); their particle was a rod-like fibre (a prolate spheroid), while for the flow field they chose the emblematic homogeneous isotropic turbulence.

For axisymmetric particles, it is convenient to decompose the angular velocity $\omega_{p}$ into spinning, $\left(\omega_{p} \cdot p\right)$, and tumbling, $\dot{p} \equiv\left(\omega_{p} \times p\right)$, components, with $p$ representing the symmetry axis unit vector, and $\left|\omega_{p}\right|=\sqrt{\left(\omega_{p} \cdot p\right)^{2}+\dot{p}^{2}}$. If the particles were to randomly orient themselves in the turbulent field, their mean-square tumbling rate can be analytically obtained as $\left\langle\dot{p}^{2}\right\rangle=1 / \tau_{\eta}^{2}\left[1 / 6+1 / 10\left(\left(\mathcal{A} \mathcal{R}^{2}-1\right) /\left(\mathcal{A} \mathcal{R}^{2}+1\right)\right)^{2}\right]$, where $\mathcal{A R}$ is the aspect ratio of the spheroid, and $\tau_{\eta}$ is the dissipative time scale (Parsa et al. 2012; Chevillard \& Meneveau 2013). However, this prediction is at striking odds with both experiments and simulations of spheroids advected through isotropic turbulence (Parsa et al. 2012; Byron et al. 2015; Ni et al. 2015). For prolate spheroids $(\mathcal{A} \mathcal{R} \gg 1)$, the measurements are smaller by nearly a factor of 3 than the predicted value $(\approx 4 / 15)$ for randomly distributed orientations.

A qualitative explanation for this disparity can be found by assessing the particle's preferential alignment with the velocity gradients of the flow. Since the velocity gradients are finite-time correlated in turbulent flows, Lagrangian stretching would align rods $(\mathcal{A R} \gg 1)$ with the vorticity $\omega_{f}$, while disks $(\mathcal{A R} \ll 1)$ tend to align their symmetry axis perpendicular to $\omega_{f}$ (Pumir \& Wilkinson 2011; Gustavsson, Einarsson \& Mehlig 2014). Yet, a simple and elegant quantitative prediction of the mean-square spinning and tumbling rates is still missing. Further, the question of how the particle's size - in relation to the size of the turbulent eddies - affects the rotational statistics awaits a deeper evaluation. Now, Pujara et al. (2021) have shed light on these issues through a careful numerical and theoretical exploration of spheroids advected by turbulence. Their results suggest the existence of an underlying scale invariance for the rotational statistics within the Lagrangian framework.

\section{Overview}

Pujara et al. (2021) tackled the problem using a two-pronged approach. On one front, the spheroid aspect ratio was varied from oblate $(\mathcal{A R} \ll 1)$ to prolate $(\mathcal{A R} \gg 1)$. Concurrently, for sampling the various scales of turbulent motion, they resorted to a 'filtered flow' approach. The filter scale $\ell$ was varied from the very small $(\ell<\eta)$ to the very large $(\ell=L)$, where $\eta$ and $L$ are the dissipative and integral scales, respectively. 
(a)

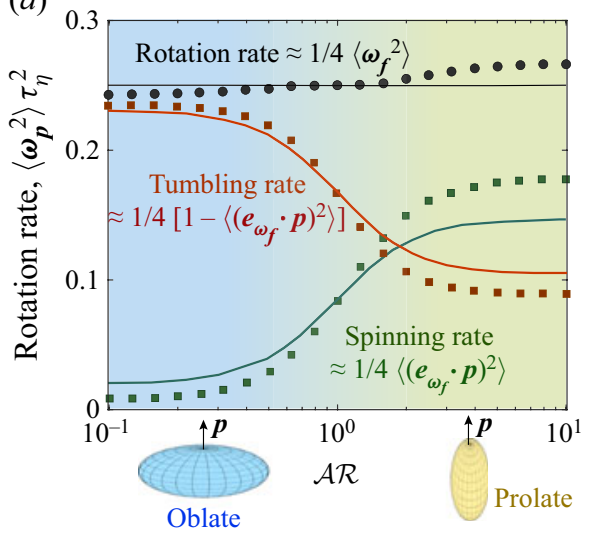

(b)

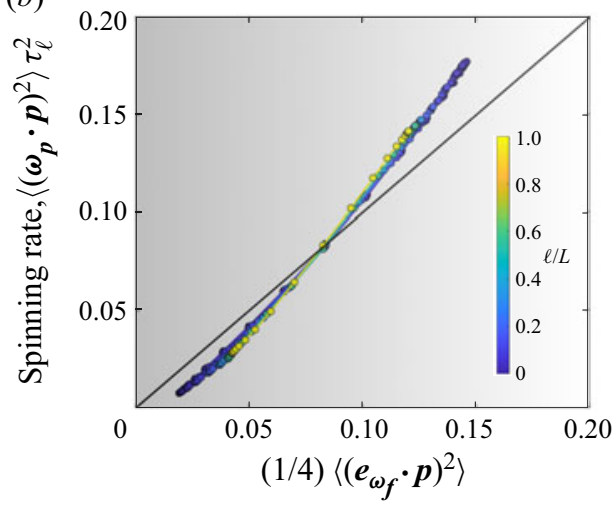

Figure 1. (a) Normalised mean-square particle angular velocity, $\left\langle\omega_{p}^{2}\right\rangle$, and its decomposition into spinning and tumbling components for spheroids in turbulence (unfiltered DNS data). (b) Nearly scale-invariant behaviour for the spinning rates for all particle shapes. In $(a, b)$, symbols are from the numerical simulations, and the solid lines give the predictions. Figures and data adapted from Pujara et al. (2021).

This allowed for quantification of how the scales of turbulent motion impact the Lagrangian statistics of particle rotation.

In agreement with previous studies (Voth \& Soldati 2017), Pujara et al. (2021) noted that the mean-square total rotation rate (MSR) of spheroids is almost independent of the particle's aspect ratio (shape invariance). With the shape invariance evident, the magnitude of the MSR can be straightforwardly deduced to be equal to $\left\langle\omega_{f}^{2}\right\rangle / 4$, because a spherical particle - which rotates identically as the fluid - lies within the range of the shape invariance. The authors elegantly derive expressions for the spinning and tumbling components of MSR by invoking one assumption - that the degree of alignment for the particle with the vorticity direction, $\boldsymbol{e}_{\omega_{f}}$, is independent of the magnitude of $\boldsymbol{\omega}_{f}$. The predicted spinning and tumbling rates for all aspect ratios $(\mathcal{A R} \in[0.1,10])$ are shown by the solid lines in figure 1(a). The simulation results - obtained by integrating Jeffery's equation along the Lagrangian trajectories - are in reasonable agreement with the authors' predictions. Deviations are surely noticeable, but it may be worthwhile to note that Pujara et al. (2021) have managed to reduce a seven-parameter problem (Ni et al. 2015) into one that depends only on the alignment between the particle and the vorticity vector.

The turbulent field considered in figure 1(a) is fully resolved; note that the normalisation time scale used is $\tau_{\eta}$. If a spatial filter of intermediate scale $\ell$ is applied to this flow field, not surprisingly the filtered flow would smooth out the fluctuations and hence the spinning and tumbling rates would be suppressed as compared with the fully resolved DNS case (unfiltered case). However, Pujara et al. (2021) provide a set of convincing arguments that the magnitude of these suppressed rotations is captured by considering the scale-dependent time scale $\tau_{\ell} \sim \ell^{2 / 3}$. Figure $1(b)$ provides a demonstration of this nearly scale-invariant rotational behaviour for particles of all aspect ratios. This scaling (first introduced by Parsa \& Voth (2014) for rods in turbulence) has found good use in a variety of settings (Mathai et al. 2016; Pujara et al. 2018). And now, the authors have extended its applicability to scales spanning the entire spatial extent of the turbulent flow - from the Kolmogorov scale to the integral scale - and across many particle geometries. 


\section{Mathai}

\section{Outlook}

The filtered flow approach by Pujara et al. (2021) might offer some insights about the ways in which finite-sized anisotropic particles respond to the scales of turbulent motion. However, it is crucial to note that the analyses here rely on the assumption (of Jeffery's equation) that the scales of motion in the flow are large compared with the particle's size. Furthermore, finite-sized particles likely deviate from the trajectories of fluid elements. The resulting slipping motion is certain to induce flow modifications that necessitate a reformulation of the torques acting on the particle (Voth \& Soldati 2017; Pujara et al. 2018).

The conclusions by Pujara et al. (2021) also hint at the possibility that fully resolved simulations may not be necessary to compute the rotational statistics of anisotropic particles. This would enable a coarse-grained simulation approach (such as large eddy simulations or truncated DNS) to sufficiently capture the rotational statistics even for sub-filter-scale particles. Its applicability though is likely to be limited to the mean-square rotation rates because quantities such as the particle's angular accelerations or the higher moments of its rotation - reflective of the rare events in turbulence - are unlikely to be scale invariant (Zimmermann et al. 2011). It is also our belief that the observed power law scalings in Pujara et al. (2021) would benefit from future explorations at higher Reynolds numbers - a wider scale separation could provide a more rigorous test of the scale invariance.

The rotational statistics observed by Pujara et al. (2018) require a closer inspection in the context of particulate flows (Voth \& Soldati 2017). As the authors carefully note, the scale invariance might only be indicative of the trends to be expected for realistic particles. Indeed, deviations are sure to arise when the effects of particle size or density or buoyancy or rotational diffusion become dominant. Despite all of these, the results of Pujara et al. (2021) have provided a glimpse into some of the underlying simplicity of the Lagrangian rotational statistics for a system that is governed by complex shape- and scale-dependent couplings between the particle and the turbulent velocity gradients. In this regard, it is our hope that this work will stimulate the development of similarly elegant models with a wider scope of applicability.

Declaration of interests. The author reports no conflict of interest.

\section{REFERENCES}

Byron, M., Einarsson, J., Gustavsson, K., Voth, G., Mehlig, B. \& Variano, E. 2015 Shape-dependence of particle rotation in isotropic turbulence. Phys. Fluids 27 (3), 035101.

Chevillard, L. \& Meneveau, C. 2013 Orientation dynamics of small, triaxial-ellipsoidal particles in isotropic turbulence. J. Fluid Mech. 737, 571-596.

Guasto, J.S., Rusconi, R. \& Stocker, R. 2012 Fluid mechanics of planktonic microorganisms. Annu. Rev. Fluid Mech. 44, 373-400.

Gustavsson, K., Einarsson, J. \& Mehlig, B. 2014 Tumbling of small axisymmetric particles in random and turbulent flows. Phys. Rev. Lett. 112 (1), 014501.

Jeffery, G.B. 1922 The motion of ellipsoidal particles immersed in a viscous fluid. Proc. R. Soc. Lond. A 102 (715), 161-179.

La Porta, A., Voth, G.A., Crawford, A.M., Alexander, J. \& Bodenschatz, E. 2001 Fluid particle accelerations in fully developed turbulence. Nature 409 (6823), 1017-1019.

Mathai, V., Neut, M.W.M., van Der Poel, E.P. \& Sun, C. 2016 Translational and rotational dynamics of a large buoyant sphere in turbulence. Exp. Fluids 57 (4), 1-10.

Ni, R., Kramel, S., Ouellette, N.T. \& Voth, G.A. 2015 Measurements of the coupling between the tumbling of rods and the velocity gradient tensor in turbulence. J. Fluid Mech. 766, 202-225.

Parsa, S., Calzavarini, E., Toschi, F. \& Voth, G.A. 2012 Rotation rate of rods in turbulent fluid flow. Phys. Rev. Lett. 109 (13), 134501. 
PARSA, S. \& Voth, G.A. 2014 Inertial range scaling in rotations of long rods in turbulence. Phys. Rev. Lett. 112 (2), 024501.

Pujara, N., Arguedas-Leiva, J.-A., Lalescu, C.C., Bramas, B. \& Wilczek, M. 2021 Shape- and scale-dependent coupling between spheroids and velocity gradients in turbulence. J. Fluid Mech. 922, R6.

Pujara, N., Oehmke, T.B., Bordoloi, A.D. \& Variano, E.A. 2018 Rotations of large inertial cubes, cuboids, cones, and cylinders in turbulence. Phys. Rev. Fluids 3 (5), 054605.

Pumir, A. \& Wilkinson, M. 2011 Orientation statistics of small particles in turbulence. New J. Phys. 13 (9), 093030.

Shin, M. \& КосH, D.L. 2005 Rotational and translational dispersion of fibres in isotropic turbulent flows. J. Fluid Mech. 540, 143-173.

Voth, G.A. \& Soldati, A. 2017 Anisotropic particles in turbulence. Annu. Rev. Fluid Mech. 49, 249-276.

Zimmermann, R., Gasteuil, Y., Bourgoin, M., Volk, R., Pumir, A. \& Pinton, J.-F. 2011 Rotational intermittency and turbulence induced lift experienced by large particles in a turbulent flow. Phys. Rev. Lett. $106(15), 154501$. 